\title{
How to Find the Lax Pair from the Yang-Baxter Equation $\star$
}

\section{Q. Zhang}

Courant Institute of Mathematical Sciences, New York University, 251 Mercer Street, New York, NY 10012, USA

Received October 25, 1990; in revised form February 25, 1991

\begin{abstract}
We show explicitly how to construct the quantum Lax pair from the Yang-Baxter equation. We demonstrate the new method by applying it to the Heisenberg XYZ model.
\end{abstract}

\section{Introduction}

It is well-known that the Yang-Baxter equations (YBE) play a crucial role in classical and quantum integrable systems (see e.g. $[1,2])$. The structure and utility of the classical YBE is now fairly well understood $[3,4]$. Unfortunately, it is much less so for its quantum partner. Although fruitful interactions between $(1+1)$ dimensional quantum field theory and $2 d$ classical mechanics have led Faddeev, Sklyanin and Takhtajan to propose the quantum inverse scattering method (QISM) $[5,6]$ as a unified extension of the classical integrable models (soliton theory) to the quantum case, the exact relation between the $R$-matrix and the Lax pair [7] is still not clear.

Consider an operator version of an auxiliary linear problem [8],

$$
\Psi_{n+1}=L_{n}(u) \Psi_{n}, \quad \dot{\Psi}_{n}=P_{n} \Psi_{n},
$$

where $L_{n}(u)$ and $P_{n}$ are matrix operators, $u$ is the spectral parameter and a dot signifies a time derivative. The consistency condition for (1) with $\dot{u}=0$ yields the Lax pair equation

$$
\dot{L}_{n}=P_{n+1} L_{n}-L_{n} P_{n} .
$$

All the solved integrable models appear to imply that "a model is completely integrable if we can find a Lax pair $\left\{L_{n}, P_{n}\right\}$ such that the Lax equation (2) is equivalent to the equation of motion of the model" [8]. One may raise a serious

* Supported by DOE contract DE-FG02-88ER25053 
question, even though a Lax pair ensures the existence of infinite conserved quantities, in principle they may not be involutive (mutually commuting). For classical integrable systems, the theory of dual symplectic (Poisson bracket) structure [10] indicates that, to prove involution, one needs additional structures on the symplectic manifold: vanishing of the Nijenhuis torsion tensor [9] (a widely studied tensor in connection with complex manifolds and analogous to the spin torsion tensor in an ordinary Riemannian manifold). On the other hand, YBE is indeed sufficient for integrability (see text). As the Lax pair formulation lies in the heart of the spectral deformation theory [11], it is therefore extremely important to know under what condition a quantum Lax pair is really equivalent to YBE. In this paper, we take the modest step to show how to compute a Lax pair from YBE. We should mention that Ganoulis [12] has constructed quantum Lax pairs for Toda systems by directly replacing the fundamental Poisson bracket [6] by a commutator, as remarked by the author that his quantization ansatz may be special to the particular system, since there the $L$-operator is homogeneous and the Hamiltonian is simply $\operatorname{tr} L^{2}$. Sogo and Wadati [13] have obtained a quantum Lax pair for the Heisenberg XYZ model through "lengthy calculations," which amount to inverting the Lax equation. Here we show a simple method to find a Lax pair systematically by using the YBE and the $\mathrm{XYZ}$ model as a working example.

A brief review of the YBE and its connection to different physical branches is given in Sect. 2. In Sect. 3, by making use of the infinitesimal version of the local YBE, we derive a formula for calculating Lax pairs. In Sect. 4, we apply the method to the $\mathrm{XYZ}$ model and compare the result with the previous calculation. Finally we make a few remarks about future research in the last section.

\section{Yang-Baxter Equation and Its Local Forms}

Starting with a local $L$-operator $L_{n}$ of the QISM (it is a matrix which acts in $V$ and has elements which act in $h_{n}$, where $V$ is the auxiliary space and $h_{n}$ the quantum space at site $n$ ), we can form a transfer matrix $T_{N}(u)$ (see e.g. [14])

$$
T_{N}(u)=\operatorname{Tr} \mathscr{T}_{N}(u)
$$

where the monodromy matrix $\mathscr{T}_{N}(u)$ is given by

$$
\mathscr{T}_{N}(u)=L_{N}(u) L_{N-1}(u) \cdots L_{2}(u) L_{1}(u)
$$

we assume a periodic chain. It is obvious that $T_{N}(u)$ is time independent and therefore can be used as a generator of the conserved quantities. (The method of extracting the local integrals of motion from the quantum monodromy matrix $T_{N}(u)$ is known as a trace formulae [15].) For most quantum integrable systems, we can show that direct products of two $L$-operators with different spectral parameters satisfy a similarity relation

$$
R(u-v)\left[L_{n}(u) \otimes L_{n}(v)\right]=\left[L_{n}(v) \otimes L_{n}(u)\right] R(u-v),
$$

with $R$ a $c$-number matrix acting in $V \times V$. The above relation is referred to as (local) YBE and can be represented graphically by Fig. 1. 

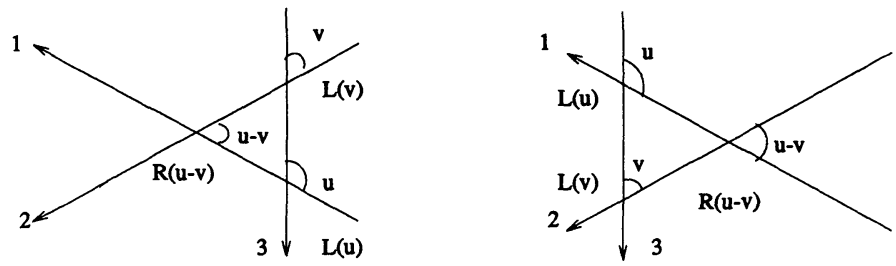

Fig. 1

If $L_{n}$ 's with different $n$ commute, we further have

$$
R(u-v)\left[\mathscr{T}_{N}(u) \otimes \mathscr{T}_{N}(v)\right]=\left[\mathscr{T}_{N}(v) \otimes \mathscr{T}_{N}(u)\right] R(u-v),
$$

called global YBE. Note that (6) is also valid for continuous models. The physical significance of the YBE is that it implies the existence of an infinite number of conserved quantities that are involutive. This can be easily checked by taking the trace on both sides of (6),

$$
\left[T_{N}(u), T_{N}(v)\right]=0 .
$$

And off-diagonal elements of (6) offer an algebraic formulation of the Bethe ansatz method $[14,16]$.

We assume the auxiliary space $V$ and the quantum space $h$ are the same and regard the elements of $L$ and $R$ as those of the scattering matrix ( $S$-matrix), the YBE equation (5) may then be written as

$$
S_{12}(u-v) S_{13}(u) S_{23}(v)=S_{23}(v) S_{13}(u) S_{12}(u-v),
$$

where $S_{i j}$ is a matrix in $V \times V \times V$, which acts nontrivially in spaces $V_{i}$ and $V_{j}$ only. The spectral parameter $u$ represents the rapidity-difference in the two particle scattering on a rotated [17] lattice (see Fig. 1). This relation is called the factorization equation because it implies the scattering theory is factorizable. It is interesting to note that (8) is interpreted as the condition for associativity of the semigroup algebra with particle creation operators $A_{k}^{\dagger}(u)$ as the generators [18]

$$
A_{i}^{\dagger}(u) A_{j}^{\dagger}(v)=S_{j k}^{i l}(u-v) A_{k}^{\dagger}(v) A_{l}^{\dagger}(u) .
$$

In $2 d$ classical statistical mechanics, $S_{j k}^{i l}(u)$ may be considered as vertices of a vertex model. Baxter first noticed the importance of this relation (8) also called the triangle equation in that context, and regarded it as the solvability condition of the vertex models [19]. Baxter also showed that the 8-vertex model is equivalent to the XYZ model [20], implying that there always exists an integrable spin model corresponding to a solvable vertex model. Zomolodchikov then pointed out that the factorization equation for the $Z_{4}$ model is essentially the same as the YBE for the 8-vertex model [21].

We see that through the YBE, the QISM set a unified framework for studying solvable models in $(1+1)$-dimensional quantum field theory, $2 d$ classical mechanics and $1 d$ quantum spin chains. They share a common property that they have the commuting "transfer matrices" and the underlying key concept is the YBE. This prompted us to research for Lax pair structures out of the YBE. 


\section{Explicit Solution for Lax Pairs (Main Result)}

To derive a Lax pair, it is convenient to use an infinitesimal version of the YBE. First we make a basic assumption: under the standard normalization (i.e. $R(0)=I$ ),

$$
R(\varepsilon) \sim I+\varepsilon\left(\alpha^{-1} H+\beta I\right)+O\left(\varepsilon^{2}\right), \quad \text { for small } \varepsilon,
$$

with $\alpha$ and $\beta$ some constants; and the Hamiltonian is a local sum given by

$$
\mathscr{H}=\sum H_{n, n-1}=\left.\alpha \frac{d}{d u} \ln T_{N}(u)\right|_{u=0},
$$

where $H_{n, n-1}$ is the $H$ in (10) acting on the quantum spaces at sites $n$ and $n-1$. This is the case for most of the models $[22,8]$ as can be checked directly (see also [23]).

We then consider the YBE shown in Fig. 2, where the spectral parameters of the local $L$-operators differ only by an infinitesimal amount $\varepsilon$. This implies that the third vertex must have parameter $\varepsilon$. A similar graph was also considered previously by Thacker [24] in the study of the Lorentz boost property of the corner transfer matrices. Note that this application of YBE is somewhat different than that which arises in the QISM. The vertices are being commuted horizontally rather than vertically and the " $R$-matrix" is not a matrix but a two-spin operator (i.e. $R=\mathscr{P} S$ with $\mathscr{P}$ the permutation operator which interchanges spaces of $V_{n}$ and $\left.V_{n-1}\right)$. By expanding in powers of $\varepsilon$,

$$
L_{n}(u+\varepsilon) \sim L_{n}(u)+\varepsilon L_{n}^{\prime}(u)+O\left(\varepsilon^{2}\right),
$$

where a prime stands for $u$-derivative. Together with (10), we find from (5) the following commutation relation:

$$
\alpha^{-1}\left[H_{n, n-1}, L_{n}(u) L_{n-1}(u)\right]=L_{n}(u) L_{n-1}^{\prime}(u)-L_{n}^{\prime}(u) L_{n-1}(u) .
$$

We multiply out the above equation and arrive at

$$
\begin{aligned}
& {\left[H_{n, n-1}, L_{n}\right]=\alpha\left(L_{n} L_{n-1}^{\prime} L_{n-1}^{-1}-L_{n}^{\prime}\right)-L_{n}\left[H_{n, n-1}, L_{n}\right] L_{n-1}^{-1},} \\
& {\left[H_{n+1, n}, L_{n}\right]=\alpha\left(L_{n}^{\prime}-L_{n+1}^{-1} L_{n+1}^{\prime} L_{n}\right)-L_{n+1}^{-1}\left[H_{n+1, n}, L_{n+1}\right] L_{n},}
\end{aligned}
$$

where we have made use of the nonsingular property of the $L$-operators.
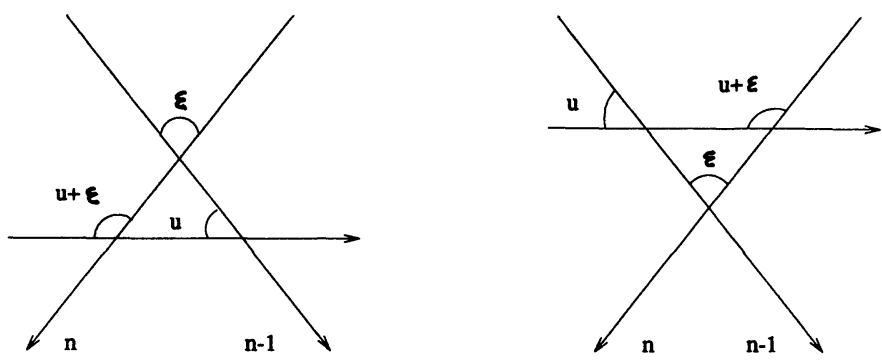

Fig. 2 
The equation of motion for $L_{n}$ is the Heisenberg equation

$$
\dot{L}_{n}=i\left[\mathscr{H}, L_{n}\right]=i\left[H_{n+1, n}, L_{n}\right]+i\left[H_{n, n-1}, L_{n}\right] \text {, }
$$

here we have set $\hbar=1$. The second equality is because of the locality of the $\mathscr{H}$ and $L_{n}$. Combining (14), (15) and (16), we have

$$
\dot{L}_{n}=-i L_{n+1}^{-1}\left\{\alpha L_{n+1}^{\prime}+\left[H_{n+1, n}, L_{n+1}\right]\right\} L_{n}+i L_{n}\left\{\alpha L_{n-1}^{\prime}-\left[H_{n, n-1}, L_{n-1}\right]\right\} L_{n-1}^{-1} \text {. }
$$

By comparing with the Lax equation (2), we can read off the other Lax operator

or

$$
P_{n}=-i L_{n}^{-1}\left\{\alpha L_{n}^{\prime}+\left[H_{n, n-1}, L_{n}\right]\right\},
$$

$$
P_{n}=-i\left\{\alpha L_{n-1}^{\prime}-\left[H_{n, n-1}, L_{n-1}\right]\right\} L_{n-1}^{-1} \text {. }
$$

The compatibility of the two expressions above are again guaranteed by the commutation relation (13) as can be checked readily. Equation (18) thus provides us with the explicit solution for a quantum Lax pair.

To summarize: for every solution $S$ of the factorization equation, we can read off $L_{n}$ and $R$ operators; if $\mathscr{H}$ has the form of (11) and related $R$ by (10), then the corresponding $P_{n}$ operator is given by (18). In the next section, we shall apply this method to a concrete model - the XYZ model.

\section{Quantum Lax Pair for the Heisenberg XYZ Model}

This model consists of a one-dimensional spin $1 / 2$ chain with the following Hamiltonian:

$$
\mathscr{H}=-\frac{1}{2} \sum J_{1} \sigma_{n}^{1} \sigma_{n-1}^{1}+J_{2} \sigma_{n}^{2} \sigma_{n-1}^{2}+J_{3} \sigma_{n}^{3} \sigma_{n-1}^{3} \equiv \sum H_{n, n-1} .
$$

The Pauli spin operators $\sigma_{n}^{j}(j=1,2,3)$ satisfy the commutation relations

$$
\begin{aligned}
{\left[\sigma_{n}^{j}, \sigma_{m}^{k}\right] } & =2 i \varepsilon^{j k l} \sigma_{n}^{l} \delta_{n m}, \\
\sigma_{n}^{j} \sigma_{n}^{k} & =\delta_{j k}+i \varepsilon^{j k l} \sigma_{n}^{l},
\end{aligned}
$$

where $\varepsilon^{j k l}$ is the antisymmetric tensor with $\varepsilon^{123}=1$. The special case $J_{1}=J_{2}$ $\left(J_{1}=J_{2}=J_{3}\right)$ is commonly referred to as XXZ (XXX) model.

From the solution of the triangle equation for the 8-vertex model [19] and the equivalence of 8-vertex model to the XYZ model [20], we know the scattering operator $S(u)$ can be written as [14]

$$
S(u)=w_{4} I+\sum_{j=1}^{3} w_{j}(u) \sigma^{j} \otimes \sigma^{j},
$$

where the coefficients are parametrized by the Jacobian elliptic functions of $u$ and modulus $l$ [25],

$$
\begin{array}{ll}
w_{1}=c n(u+\zeta, l) / c n(\zeta, l), & w_{2}=d n(u+\zeta, l) / d n(\zeta, l), \\
w_{3}=1, & w_{4}=\operatorname{sn}(u+\zeta, l) / \operatorname{sn}(\zeta, l),
\end{array}
$$

where $\zeta$ is a constant parameter (we shall suppress the modulus $l$ in what follows). 
Therefore

and

$$
L_{n}(u)=\left(\begin{array}{cc}
w_{4}+w_{3} \sigma_{n}^{3} & w_{1} \sigma_{n}^{1}-i w_{2} \sigma_{n}^{2} \\
w_{1} \sigma_{n}^{1}+i w_{2} \sigma_{n}^{2} & w_{4}-w_{3} \sigma_{n}^{3}
\end{array}\right)
$$

$$
R(u)=\mathscr{P} S(u) \quad \text { with } \quad \mathscr{P}=I+\sum_{j=1}^{3} \sigma^{j} \otimes \sigma^{j} .
$$

They satisfy the YBE (5). It follows that the transfer matrix $T_{N}(u)$ defined by (3) is the generator of the involutive conserved quantities (11). In particular, the Hamiltonian itself is given by the log-derivative [20],

and

$$
\mathscr{H}=-\left.J_{3} \operatorname{sn}(2 \zeta) \frac{d}{d u} \ln T_{N}(u)\right|_{u=0}+\text { const } I,
$$

if

$$
H=-J_{3} \operatorname{sn}(2 \zeta) R^{\prime}(0)+\text { const } I
$$

$$
J_{1}: J_{2}: J_{3}=\operatorname{cn}(2 \zeta): \operatorname{dn}(2 \zeta): 1 \text {. }
$$

Comparing these with (10) and (11), we find

$$
\alpha=-J_{3} \operatorname{sn}(2 \zeta)
$$

for this model.

To calculate $P_{n}$, we have to evaluate $L_{n}^{-1}$ which is given by

$$
L_{n}^{-1}(u)=\left(\begin{array}{cc}
v_{4}+v_{3} \sigma_{n}^{3} & v_{1} \sigma_{n}^{1}-i v_{2} \sigma_{n}^{2} \\
v_{1} \sigma_{n}^{1}+i v_{2} \sigma_{n}^{2} & v_{4}-v_{3} \sigma_{n}^{3}
\end{array}\right)
$$

where

$$
v_{1}=\frac{w_{1}\left(w_{2}^{2}+w_{3}^{2}+w_{4}^{2}-w_{1}^{2}\right)+2 w_{2} w_{3} w_{4}}{\Delta}
$$

with

$$
\Delta=\left(w_{1}+w_{2}+w_{3}-w_{4}\right)\left(w_{1}+w_{2}-w_{3}+w_{4}\right)\left(w_{1}-w_{2}+w_{3}+w_{4}\right)\left(-w_{1}+w_{2}+w_{3}+w_{4}\right),
$$

and other $v_{i}$ 's can be gotten by cyclic permutations of the indices (1234).

The next step is to calculate $L_{n}^{\prime}(u)$. For that we would have to use the properties of the Jacobian elliptic functions:

$$
s n^{\prime}(u)=c n(u) d n(u), \quad c n^{\prime}(u)=-s n(u) d n(u), \quad d n^{\prime}(u)=-l^{2} \operatorname{sn}(u) c n(u) ;
$$

or equivalently to use

$$
w_{1}^{\prime}=w_{2} w_{4} w_{1}^{\prime}(0), \quad w_{2}^{\prime}=w_{1} w_{4} w_{2}^{\prime}(0), \quad w_{3}^{\prime}=0, \quad w_{4}^{\prime}=w_{1} w_{2} w_{4}^{\prime}(0)
$$

and

$$
\begin{aligned}
& \operatorname{sn}^{2}(\zeta)=\frac{w_{1}^{2}-w_{3}^{2}}{w_{1}^{2}-w_{4}^{2}} \quad c n^{2}(\zeta)=\frac{w_{3}^{2}-w_{4}^{2}}{w_{1}^{2}-w_{4}^{2}} \\
& d n^{2}(\zeta)=\frac{w_{3}^{2}-w_{4}^{2}}{w_{2}^{2}-w_{4}^{2}} \quad l^{2}=\frac{\left(w_{1}^{2}-w_{4}^{2}\right)\left(w_{2}^{2}-w_{3}^{2}\right)}{\left(w_{1}^{2}-w_{3}^{2}\right)\left(w_{2}^{2}-w_{4}^{2}\right)}
\end{aligned}
$$


But in fact, due to the tensor properties of the spin model, we do not have to calculate $L_{n}^{\prime}(u)$ at all. Since the general form of $P_{n}$ has to be [13]

$$
P_{n}=\left(\begin{array}{cc}
p_{n}^{4}+p_{n}^{3} \sigma_{n}^{3} & p_{n}^{1} \sigma_{n}^{1}-i p_{n}^{2} \sigma_{n}^{2} \\
p_{n}^{1} \sigma_{n}^{1}+i p_{n}^{2} \sigma_{n}^{2} & p_{n}^{4}-p_{n}^{3} \sigma_{n}^{3}
\end{array}\right)
$$

where

$$
\begin{aligned}
& p_{n}^{4}=\sum_{j=1}^{3} A_{j} \sigma_{n}^{j} \sigma_{n-1}^{j}+\text { const } I, \\
& p_{n}^{i}=B_{i}\left(\sigma_{n}^{i}+\sigma_{n-1}^{i}\right)+\sum_{j=1}^{3} \sum_{k=1}^{3} \varepsilon^{i j k} C_{i} \sigma_{n}^{j} \sigma_{n-1}^{k} \quad(i=1,2,3) .
\end{aligned}
$$

To determine the coefficients $A_{k}, B_{k}, C_{k},(k=1,2,3)$, we only need to calculate the second term in (18),

$$
i L_{n}^{-1}\left[H_{n, n-1}, L_{n}\right]=-\left(v_{4}+\sum_{j=1}^{3} v_{j} \sigma^{j} \sigma_{n}^{j}\right) \sum_{j, k, l=1}^{3} \varepsilon^{j k l} J_{k} w_{l} \sigma_{n}^{j} \sigma_{n-1}^{k} \sigma^{l},
$$

which is proportional to $\sigma_{n-1}$.

Even better, we only have to calculate the coefficient of $\sigma_{n-1}^{1}$,

$$
\begin{aligned}
& -i J_{1}\left(v_{2} w_{2}+v_{3} w_{3}\right) \sigma_{n}^{1} \sigma^{1}+i J_{1}\left(v_{2} w_{3}+v_{3} w_{2}\right) \sigma^{1} \\
& \quad+J_{1} v_{1}\left(w_{3} \sigma_{n}^{3} \sigma^{2}-w_{2} \sigma_{n}^{2} \sigma^{3}\right)-J_{1} v_{4}\left(w_{2} \sigma_{n}^{3} \sigma^{2}-w_{3} \sigma_{n}^{2} \sigma^{3}\right) .
\end{aligned}
$$

Comparing (34) with (32), we find

$$
\begin{aligned}
& A_{1}=i J_{1} \frac{\left(w_{1}^{2}+w_{2}^{2}-w_{3}^{2}-w_{4}^{2}\right)\left(w_{2}^{2}+w_{4}^{2}-w_{1}^{2}-w_{3}^{2}\right)-\Delta}{2 \Delta}, \\
& B_{1}=2 i J_{1} \frac{w_{1} w_{4}\left(w_{2}^{2}+w_{3}^{2}\right)+w_{2} w_{3}\left(w_{1}^{2}+w_{4}^{2}\right)}{\Delta}, \\
& C_{2}=J_{1} \frac{\left(w_{1} w_{3}+w_{2} w_{4}\right)\left(w_{3}^{2}+w_{4}^{2}-w_{1}^{2}-w_{2}^{2}\right)}{\Delta},
\end{aligned}
$$

where $\Delta$ is defined in (30). Again, other coefficients may be obtained by the cyclic permutation (123). We see that up to a diagonal term which is always arbitrary, the solution is exactly the same as those obtained by direct computations in [13].

\section{Final Remarks}

In this paper, we have derived a Lax pair based on a particular local Hamiltonian which is the first log-derivative of the partition function and has only nearest neighbor interactions. It is easy to see that one can generalize it immediately to other local Hamiltonians defined by higher derivatives of $\ln T_{N}(u)$ (Lüscher has calculated these for the XYZ model and showed that they are local-each derivative only introduces one further neighboring interaction). Of course, one then has to use an appropriate commutation relation obtained by expanding $S(u)$ 
to higher order. This way, one arrives at a family of Lax pairs corresponding to a family of involutive Hamiltonians, which is rather similar to the classical case where one finds a whole family of commuting Hamiltonian flows moving along the geodesic curves of the symplectic manifold (and the gauge group can be expressed in terms of theta functions on the Jacobi varieties of the curves [26]).

The other half of the question still remains: what properties, in addition to a Lax pair, one might need in order to derive the YBE? We hope to answer this question in the future. In any case, it is already pretty obvious that the $R$ (or $S$ )-matrix which is the solution of the YBE plays a central role in the whole construct. For each $S$-matrix, there exists a family of Lax pairs corresponding to a family of involutive Hamiltonians and a $L$-operator which is taken to be the $S$ itself. Of course, there can be other $L$-operators, hence a different family of Lax pairs. But we expect that these are essentially related by local gauge transformations (i.e. new $L$-operator is given by $U_{n+1} L_{n} U_{n}^{-1}$ for some $U_{n}$ ). This shows the YBE is more basic than the Lax pair equation for quantum integrable systems. Therefore finding all possible solutions of the YBE and classifying all $R$-matrices are the most important issues, we hope the current intensive studies of the (Lie algebra) deformation theory [27-29] which led to the studies of the quantum group and Hopf algebra will eventually provide us the answers [30, 2, 31]. Quantized algebras may, in turn, give rise to new powerful algorithmic applications as the Lie algebras do [32].

Acknowledgements. The author would like to thank P. Deift, J. Moser, H. McKean and P. Lax for helpful discussions and references.

\section{References}

1. Kulish, P. P., Sklyanin, E. K.: J. Sov. Math. 19, 1596 (1982)

2. Jimbo, M.: Introduction to the Yang-Baxter equation. In: Braid Group, Knot Theory and Statistical Mechanics. Yang, C. N., Ge, M. L. (eds). Singapore: World Scientific 1989

3. Semenov-Tyan-Shanskii, M. A.: What is a classical $R$-matrix? Funct. Anal. Appl. 17, 259 (1983); Drinfel'd, V. G.: Hamiltonian structures on Lie groups, Lie bi-algebras and the geometric meaning of the classical Yang-Baxter equations. Sov. Math. Dokl. 27, 68 (1983); Reshetikhin, N. Yu., Faddeev, L. D.: Hamiltonian structures for integrable models of field theory. Theor. Math. Phys. 56, 847 (1984)

4. Belavin, A. A., Drinfel'd, V. G.: Solutions of the classical Yang-Baxter equation for simple Lie algebras. Funct. Anal. Appl. 16, 159 (1982)

5. Faddeev, L. D., Sklyanin, E. K., Takhtajan, L. A.: The quantum inverse problem I. Theor. Math. Phys. 40, 194 (1979)

6. Faddeev, L. D.: Integrable models in $(1+1)$-dimensional quantum field theory. Les Houches Session XXXIX, vol 563. Amsterdam: Elsevier 1982

7. Lax, P. D.: Integrals of nonlinear equations of evolution and solitary waves. Commun. Pure. Appl. Math. 21, 467 (1968). For a more recent reference, see e.g. Kupershmidt, B. A., Wilson, G.: Modifying Lax equations and the second Hamiltonian structure. Invent. Math. 62, 403 (1981)

8. Wadati, M., Akutsu, M.: From solitons to knots and links. Prog. Theor. Phys. 94, 1 (1988)

9. Nijenhuis, A.: Indag, Math. 13, 200 (1951)

10. Magri, F.: A simple model of the integrable Hamiltonian equation. J. Math. Phys. 19, 1156 (1978); Okubo, S., Das. A: The integrabiliy condition for dynamical systems. Phys. Lett. B209, 311 (1988) 
11. Moser, J.: Three integrable Hamiltonian systems connected with isospectral deformations. Adv. Math. 16, 197 (1975)

12. Ganoulis, N.: Quantum Toda systems and Lax pairs. Commun. Math. Phys. 109, 23 (1987)

13. Sogo, K., Wadati, M.: Quantum inverse scattering method and Yang-Baxter relation for integrable spin systems. Prog. Theor. Phys. 68, 85 (1982)

14. Takhtadzhan, L. A., Faddeev, L. D.: The quantum method of the inverse problem and the Heisenberg XYZ model. Russ. Math. Surv. 34, 11 (1979)

15. Izergin, A., Korepin, V. E.: Lattice versions of quantum field theory models in two dimensions. Nucl. Phys. B205, FS5, 401 (1982)

16. Thacker, H. B.: Exact integrability in quantum field theory and statistical mechanics. Rev. Mod. Phys. 53, 253 (1981)

17. Bariev, R. Z.: Two-dimensional ice-type vertex model with two types of staggered sites. Theor. Math. Phys. 49, 1021 (1981)

18. Zamolodchikov, A. B., Zamolodchikov, Al. B: Factorized $S$-matrices in two dimensions as the exact solutions of certain relativistic quantum field theory models. Ann. Phys. 120, 253 (1979)

19. Baxter, R. J.: Partition function of the eight-vertex lattice model. Ann. Phys. 70, 193 (1972)

20. Baxter, R. J.: One-dimensional anisotropic Heisenberg chain. Ann. Phys. 70, 323 (1972)

21. Zamolodchikov, A. B.: $Z_{4}$-symmetric factorized $S$-matrix in two space-time dimensions. Commun. Math. Phys. 69, 165 (1979)

22. Jimbo, M.: Quantum $R$ matrix for the generalized Toda system. Commun. Math. Phys. 102, 537 (1986)

23. Kulish, P. P., Sklyanin, E. K.: Quantum spectral transform method, Recent developments. In: Integrable quantum field theories. Lect. Notes in Phys. vol 151. Berlin, Heidelberg, New York: Springer 1982

24. Thacker, H. B.: Corner transfer matrices and Lorentz invariance on a lattice. Physica D18, 348 (1986)

25. Gradshteyn, I. S., Ryzhik, I. M.: Tables of integrals, series and products, p. 904. New York: Academic Press 1965

26. McKean, H.: Integrable systems and algebraic curves. Lect. Notes in Math. vol 755, p. 83. Berlin, Heidelberg, New York: Springer 1979

27. Reshetikhin, N. Yu., Kulish, P. P.: The quantum linear problem for the Sine-Gordon equation and higher representations. Zapiski nauch. LOMI 101, 101 (1980)

28. Drinfel'd, V. G.: Hopf algebras and the quantum Yang-Baxter equation. Sov. Math. Dokl. 32, 254 (1985)

29. Jimbo, M.: A $q$-difference analogue of $\mathscr{U}_{q} g$ and the Yang-Baxter equation. Lett. Math. Phys. $10,63(1985)$

30. Drinfel'd, V. G.: Quantum groups. ICM Proceedings, Berkeley, p. 798 (1986)

31. Faddeev, L. D., Reshetikhin, N. Yu., Takhtajan, L. A.: Quantum groups. In: Braid Group, Knot Theory and Statistical Mechanics. Yang, C. N., Ge, M. L. (eds). Singapore: World Scientific 1989

32. Deift, P. A., Li, L. C.: Generalized affine Lie algebras and the solution of a class of flows associated with the QR eigenvalue algorithm. Preprint (1990); Deift, P. A., Li, L. C., Tomei, C.: Matrix factorization and integrable systems. Commun. Pure Appl. Math. XLII, 443(1989)

Communicated by M. Aizenman 
